\title{
Experiments on Passive Hypervelocity Boundary-Layer Control Using an Ultrasonically Absorptive Surface
}

\author{
A. Rasheed* and H. G. Hornung ${ }^{\dagger}$ \\ California Institute of Technology, Pasadena, California 91125 \\ A. V. Fedorov \\ Moscow Institute of Physics and Technology, Zhukovski, 140160, Russia \\ and \\ N. D. Malmuth ${ }^{\S}$ \\ Rockwell Science Center, Thousand Oaks, California 91630
}

\begin{abstract}
Recently performed linear stability analyses suggested that transition could be delayed in hypersonic boundary layers by using an ultrasonically absorptive surface to damp the second mode (Mack mode). Boundary-layer transition experiments were performed on a sharp 5.06-deg half-angle round cone at zero angle of attack in the T5 Hypervelocity Shock Tunnel to test this concept. The cone was constructed with a smooth surface around half the cone circumference (to serve as a control) and an acoustically absorptive porous surface on the other half. Test gases investigated included nitrogen and carbon dioxide at $M_{\infty} \simeq 5$ with specific reservoir enthalpy ranging from 1.3 to $13.0 \mathrm{MJ} / \mathrm{kg}$ and reservoir pressure ranging from 9.0 to $50.0 \mathrm{MPa}$. Comparisons were performed to ensure that previous results obtained in similar experiments (on a regular smooth surface) were reproduced, and the results were extended to examine the effects of the porous surface. These experiments indicated that the porous surface was highly effective in delaying transition provided that the pore size was significantly smaller than the viscous length scale.
\end{abstract}

$\quad$ Nomenclature
$h=$ enthalpy, $\mathrm{MJ} / \mathrm{kg}$
$M=$ Mach number
$P=$ pressure, $\mathrm{MPa}$
$\dot{q}=$ heat flux, $\mathrm{MW} / \mathrm{m}^{2}$
$R e=$ Reynolds number
$S t=$ Stanton number
$\rho=$ density, $\mathrm{kg} / \mathrm{m}^{3}$
$\mu \quad=$ viscosity, $\mathrm{N} \cdot \mathrm{s} / \mathrm{m}^{2}$

\section{Subscripts}

aw $=$ adiabatic wall

$D=$ hole diameter

$e \quad=$ edge condition

$k=$ roughness height

$s=$ slot width

$\operatorname{tr}=$ transition

$x=$ location on cone measured along surface, $\mathrm{mm}$

$0=$ stagnation condition

$\infty=$ freestream

Superscript

* $\quad=$ reference condition

Presented as Paper 2001-0274 at the 39th Aerospace Sciences Meeting, Reno, NV, 8-11 January 2001; received 16 February 2001; revision received 4 September 2001; accepted for publication 4 September 2001. Copyright (C) 2001 by the authors. Published by the American Institute of Aeronautics and Astronautics, Inc., with permission. Copies of this paper may be made for personal or internal use, on condition that the copier pay the $\$ 10.00$ per-copy fee to the Copyright Clearance Center, Inc., 222 Rosewood Drive, Danvers, MA 01923; include the code 0001-1452/02 \$10.00 in correspondence with the CCC.

* Graduate Research Assistant, Graduate Aeronautical Laboratories. Student Member AIAA.

${ }^{\dagger}$ Clarence L. Johnson Professor of Aeronautics, Director of Graduate Aeronautical Laboratories. Member AIAA.

*Associate Professor, Department of Aeromechanics and FlightEngineering. Member AIAA.

${ }^{\S}$ Senior Scientist, Fluid Dynamics. Fellow AIAA.

\section{Introduction}

$\mathbf{H}$ EAT loads and forces on hypervelocity vehicles depend critically on the location of transition from laminar to turbulent flow. Although significant progress has been and is being made in the theoretical understanding of transition, it is still one of the most severe uncertainties in the aerodynamic design of such vehicles.

An extensive series of experiments studying boundary-layertransition over a 5-deg smooth surfaced cone has previously been performed in the T5 Hypervelocity Shock Tunnel by Germain and Hornung ${ }^{1}$ and Adam and Hornung. ${ }^{2}$ In addition to capturing a flow visualization (shadowgraph) image of the boundary-layertransition process, these experiments determined the dependence of transition Reynolds number on specific stagnation enthalpy. One of the main results obtained by Germain and Hornung ${ }^{1}$ was that the transition Reynolds number correlated with specific stagnation enthalpy provided that the Reynolds number was calculated at a reference temperature ${ }^{3}$ rather than the boundary-layer edge conditions. As shown by Adam and Hornung, ${ }^{2}$ the reference condition characterizes the conditions in the boundary layer better than the edge conditions, especially when comparisons are made between flows at different Mach numbers. These experiments also observed a trend of delayed transition as enthalpy increased.It is known that acoustic waves are absorbed by chemical activity, and the delayed transition was attributed to the increased chemical activity acting as a damping mechanism on the growth rate of the second mode acoustic instability waves.

In the absence of large freestream disturbances or surface perturbations, transition is caused by the amplification of unstable boundary-layermodes. ${ }^{4}$ At hypersonicconditions $(M \gtrsim 4)$, the dominant instabilitymode in two-dimensionalor quasi-two-dimensional boundary layers is the so-called second mode or Mack mode. ${ }^{5}$ In this mode, freestream acoustic perturbations become trapped in the boundary layer, grow in amplitude, and eventually cause the boundary layer to become turbulent. The most strongly amplified wavelength of these acoustic perturbations is approximately twice the boundary-layer thickness and propagates with a phase velocity approximately equal to the boundary-layer edge velocity. ${ }^{6}$ This is entirely different from lower speed flows, where transition is dominated by the Tollmien-Schlichting mode. Linear stability computations by Johnson et al. ${ }^{7}$ indicated that, at T5 tunnel experimental 
conditions, the most unstable mode had frequencies of the order of 1-3 MHz. Such high frequencies are highly indicative of the second mode and confirmed that the Mack mode was indeed the most strongly amplified mode. These computations also confirmed the experimental observation that increased chemical activity at higher enthalpies damped the second mode growth rates and delayed transition at the T5 tunnel conditions.

Even more desirable than being able to predict the transition location would be to control it, specifically to move it downstream. Inviscid and viscous second mode stability analyses recently performed by Fedorov and Malmuth ${ }^{8}$ and Fedorov et al. ${ }^{9,10}$ suggested that transition could be delayed in hypersonic flow by using a suitably porous surface that would absorb and dissipate the most unstable second mode acoustic waves. The porous surface considered in the analysis was a surface pitted by equally spaced cylindricalblind microholes, that is, holes with closed bottoms, whose size and spacing were determined by the frequencies (or rather, the wavelengths) of the most unstable mode. The scale of the required porosity at the high frequencies of the second mode is extremely fine and would not prematurely trip the boundary layer by other mechanisms. Note that the proposed control mechanism is purely passive and that there is no net flow (suction or blowing) through the holes. This paper will discuss the details of the experiments performed in the T5 Hypervelocity Shock Tunnel to test the computational prediction by Fedorov et al. ${ }^{10}$ that suitable wall porosity delays transition in hypersonic boundary layers.

\section{Experimental Objective}

The main objective was to test the boundary-layercontrol scheme by testing a nominal 5-deg half-angle cone with a smooth surface on one side and the ultrasonically absorbing porous surface on the other side. In this manner, each experiment was self-contained in that the smooth surface transition Reynolds number provided a direct baseline for the porous surface results. In addition, these experiments were used to verify agreement with previous data obtained by Germain and Hornung ${ }^{1}$ and Adam and Hornung, ${ }^{2}$ to test repeatability and to confirm that nonaxisymmetry or angle-of-attackissues were not affecting the results.

The previous set of experiments and linear stability analyses provided the basis for the current experimental study. In particular, the Mack mode was identified as being dominant at T5 tunnel conditions, and the proposed control scheme addresses this mode. Furthermore, the transition data from previous experiments provided important comparison data and served as a checkpoint for the new results. Finally, Adam and Hornung's comparison ${ }^{2}$ with flight data ensured that the experimental results obtained were relevant to actual future flight vehicles.

\section{T5 Hypervelocity Shock Tunnel}

\section{Experimental Setup}

This series of experiments was performed in the Graduate Aeronautical Laboratories, California Institute of Technology, T5 Hypervelocity Free-Piston Shock Tunnel. In this reflected shock tunnel facility, a piston is initially launched by high-pressure air. The resulting adiabatic compression of a helium-argon mixture bursts a diaphragm $\left(P_{\text {burst }} \simeq 90 \mathrm{MPa}\right)$ causing a shock wave to travel into a shock tube, whose end wall is closed except for the small throat of the nozzle. The reflected shock from the end wall creates a quasiconstant pressure reservoir for the subsequent steady expansion through the nozzle. Shock speeds of up to $5 \mathrm{~km} / \mathrm{s}$ can be obtained to produce nominal Mach 5 flows with a specific reservoir enthalpy of up to $25 \mathrm{MJ} / \mathrm{kg}$, reservoir pressure of $60 \mathrm{MPa}$, and reservoir temperature of $8000 \mathrm{~K}$. Typical flow velocities for the present experiments were of the order of 3-4 km/s with typical useful test times ranging from 1 to $2 \mathrm{~ms}$. Existing shock tunnel diagnostic instrumentation provided the shock speed and reservoir pressure from which the freestream conditions were calculated. The stagnation enthalpy was calculated by solving the one-dimensionalequilibriumgasdynamics reflected shock problem using the measured shock speed, the measured initial shock tube pressure, and room temperature as inputs. The freestream conditions were calculated using a one-dimensional, inviscid, vibrational equilibrium, chemical nonequilibrium nozzle code, which used the computed stagnation temperature and measured stagnation pressure as inputs. Detailed descriptions regarding T5 operations and performance may be found in Hornung. ${ }^{11}$

\section{Model and Instrumentation}

Model Configuration

The model used for these experiments was a heavily modified version of the same model used by Germain and Hornung ${ }^{1}$ and Adam and Hornung. ${ }^{2}$ The final configuration was a sharp 5.06-deg halfangle round cone consisting of five pieces with an overall length of $999 \mathrm{~mm}$. It had a smooth surface over half the cone and the absorptive porous surface over the other half beginning at $148 \mathrm{~mm}$ from the cone tip (Fig. 1). The aluminum base cone used by Germain and Hornung ${ }^{1}$ and Adam and Hornung ${ }^{2}$ was lengthened by the cone insert for the purposes of this project. The base cone was already hollow to allow room for instrumentation, and this aspect was preserved. An intermediate piece made of stainless steel 304 referred to as the cone tip holder was screwed into this assembly. The molybdenum cone tip was then screwed into this intermediate piece and was easily replaceable in the event of excessive blunting or other damage. Extreme care was taken during the manufacturing process to minimize the steps at these junctions.

The cone sheet was manufactured by rolling two initially flat sheets of metal (one perforated, one smooth) to form two longitudinal halves of a cone and then laser fusion welding them along the seams. Refer to Fig. 2a for a micrograph of the final weld. The resulting stainless steel cone sheet was fitted over the aluminum base cone using a thermal interference fit that took advantage of the mismatch in thermal coefficients of expansion of the two materials. This was accomplished by cooling both parts down to $190 \mathrm{~K}$ in special freezers used to store biologicalcell samples. This assembly technique resulted in the cone sheet being stretched tightly over the base cone providing the blind microholes for the porous surface and eliminating any surface imperfections. In addition, it eliminated the need for any mechanical fasteners that would disturb the boundary layer. Finally, this attachment method allowed the cone sheet to be nondestructively removable (and thus reusable) by simply reversing the thermal interference fit process. Note that all surfaces that were exposed to the flow were made of stainless steel 304, with the exception of the removable tip, which was made of molybdenum.

The final fully assembled model was placed in a lathe, and indicators were used to verify the geometry of the cone. In particular, the half-angle of the cone was verified to be $5.06 \mathrm{deg}$, and the steps at the two junctions (cone tip/cone tip holder and cone tip holder/cone sheet junctions) were measured to be less than $0.038 \pm 0.006 \mathrm{~mm}$. Table 1 summarizes the angular distribution of the measured step

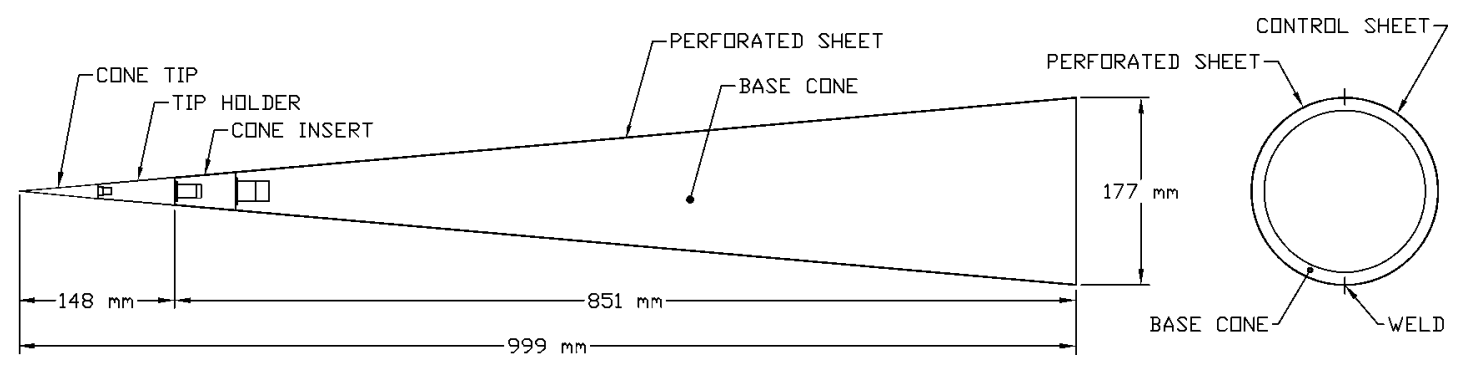

Fig. 1 Schematic diagram of the assembled test model from the profile and rear view. 
heights. This was acceptable because previous experiments by Germain and Hornung ${ }^{1}$ at similar run conditions indicated that roughnesselements, and steps as large as $0.1 \mathrm{~mm}$ placed at the $x=76$ and $203 \mathrm{~mm}$ locations had no measurable effect on the transition location.

As expected, there were highly localized imperfections along the welded seams of the cone sheet, even after the thermal interference fit assembly process. The size of the bumps was approximately constant along the length of the cone such that the local increase in diameter was about $0.3 \mathrm{~mm}$. Note that this effect was very small. The cross-sectional diameter at the front edge of the cone sheet (where the effect was most pronounced) was $26.1 \mathrm{~mm}$, resulting in the bump height being less than $1 \%$ of the local diameter. The cone tip was sharp with a measured radius of $0.076 \pm 0.005 \mathrm{~mm}$. A limited amount of blunting of the tip occurred over the course of these experiments (blunted radius of $0.130 \pm 0.005 \mathrm{~mm}$ ) due to the high heat loads at the stagnation point. Studies by Germain and Hornung $^{1}$ (and confirmed in the present work) indicated that the effect of this minimal blunting on transition Reynolds number fell well within the overall error tolerance and, therefore, was not relevant for this series of experiments. New and blunted nose tips were examined under a microscope and were observed to be perfectly straight. The most significant imperfection was a small gap that existed between the cone sheet and the base cone. This gap was localized near the beginning edge of the cone sheet and was the result of imperfect rolling/welding of the cone sheet halves. This was of concern because the gap causes suction through the perforated sheet during the time it takes the test gas to fill the cavity between the cone sheet and the base cone. This would, of course, have a strong stabilizing effect on the boundary layer. After the pressure equalizes, however, the suction effect would no longer occur. The maximum gap height was measured to be less than $0.05 \mathrm{~mm}$ and extended no more than $50 \mathrm{~mm}$ downstream on the porous side of the cone only, that is, the affected area extended from approximately 150 to $200 \mathrm{~mm}$ as measured from the cone tip along the surface. The quoted numbers are considered to be conservative estimates and result in a maximum cavity volume of $0.3 \mathrm{~cm}^{3}$. The affected

Table 1 Measured step heights ${ }^{\mathrm{a}}$ at cone tip/cone tip holder $^{\mathrm{b}}$ and cone tip holder/cone sheet ${ }^{\mathrm{c}}$ interfaces

\begin{tabular}{lcr}
\hline \hline Angle, deg & $\begin{array}{c}\text { Junction A, } \\
\mathrm{mm}\end{array}$ & $\begin{array}{c}\text { Junction B, } \\
\mathrm{mm}\end{array}$ \\
\hline 0 (weld) & 0.000 & 0.025 \\
90 (smooth surface) & 0.013 & -0.013 \\
180 (weld) & 0.013 & 0.025 \\
270 (porous surface) & 0.013 & 0.038 \\
\hline \hline
\end{tabular}

a All step height measurements are $\pm 0.006 \mathrm{~mm}$. Positive quantities indicate forward facing steps; negative quantities indicate backward facing steps; angular locations are further defined in Fig. 3.

${ }^{\mathrm{b}}$ Junction A, $x=75 \mathrm{~mm}$.

${ }^{\mathrm{c}}$ Junction B, $x=148 \mathrm{~mm}$

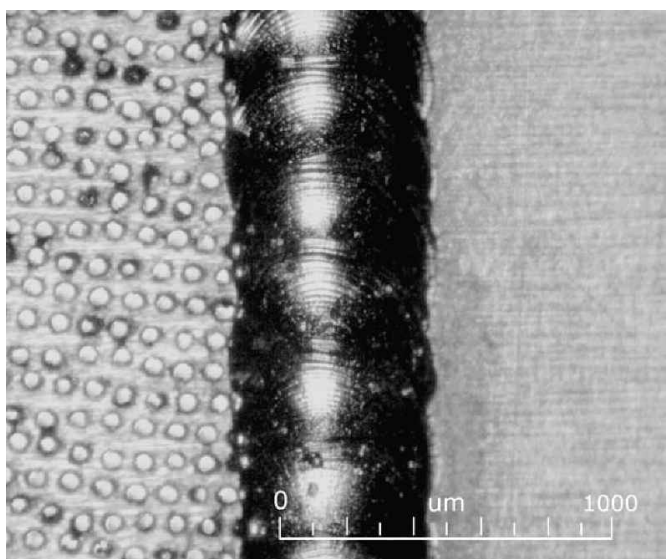

a) surface area was about $48 \mathrm{~cm}^{2}$ resulting in approximately 480,000 holes connecting the gap to the freestream. Conservative estimates with an assumed cavity volume of $1 \mathrm{~cm}^{3}$ indicated that it would take approximately $25 \mu$ s to fill the gap. This is well within the $0.5 \mathrm{~ms}$ required for the nozzle to start and for steady flow to be established. Furthermore, data were never taken earlier than $1.0 \mathrm{~ms}$ after the start of the experiment, which allowed more than sufficient time for the gap to be filled and the pressure to be equalized. Note that no flow could enter this gap in the streamwise direction because the front edge of the cone sheet at the gap location butted solidly against the back of the cone tip holder. For these reasons, it is felt that the small gap had no appreciable effect on the results.

\section{Porous Sheet}

As indicatedearlier, the very high frequencies, that is, small wavelengths, of interest necessitated extremely fine porosity. The desired parameters for the porous surface were determined based on the estimated wavelength of the most unstable mode. Numerical simulations by Adam ${ }^{12}$ indicated that the boundary-layer thickness was approximately $0.5-1 \mathrm{~mm}$ (computed at the $x=305$ and $914 \mathrm{~mm}$ locations); therefore, the most unstable mode had a wavelength of approximately 1-2 $\mathrm{mm}$. The final porous sheet had $60 \pm 4 \mu \mathrm{m}$ diameter holes spaced $100 \pm 7 \mu \mathrm{m}$ apart in a rectangular grid arrangement. This resulted in approximately 10-20 holes per disturbance wavelength and an open area (or porosity) of $28 \%$. Because of the nature of the laser drilling process, the holes were slightly conical (taper angle of about $0.5-\mathrm{deg}$ ) with the small diameter exposed to the flow. The thickness of the sheet (thus, the depth of the holes) was $450 \mu \mathrm{m}$ (26 gauge sheet) and followed the Fedorov et al. ${ }^{10}$ analysis that the depth of the holes be approximately $30 \%$ of the boundary-layer displacement thickness. The porous surface began at approximately $148 \mathrm{~mm}$ from the tip of the cone as per the Fedorov et al. analysis ${ }^{10}$ using the lower branch of the neutral stability curve for the Mack mode at a frequency of $1 \mathrm{MHz}$. Refer to Fig. $2 \mathrm{~b}$ for a micrograph of the porous surface.

\section{Instrumentation}

The model was instrumented with 56 Type E flush-mountedcoaxial thermocouples of which 52 ( 26 per side) were actually used to collect data because this was the maximum number of channels available on the data acquisition system. The thermocouples were arranged in a staggered pattern $24.5 \mathrm{~mm}$ apart with the first one located at $255 \mathrm{~mm}$ and the last one located at $814 \mathrm{~mm}$ from the tip. Figure 3 is a schematic diagram showing the location of the thermocouples on a developed view of half the cone, that is, the smooth surface side. The azimuthal lines are drawn at 12.7 -mm $(0.5$-in.) intervals with the third line located at $165 \mathrm{~mm}$ (6.5 in.). The second line shows the location of the cone tip holder/cone sheet interface at $148 \mathrm{~mm}$ (5.8 in.). The porous surface side had the mirror image of the same layout. Note that the thermocouples were deliberately placed as far from the weld as possible, with the closest one being

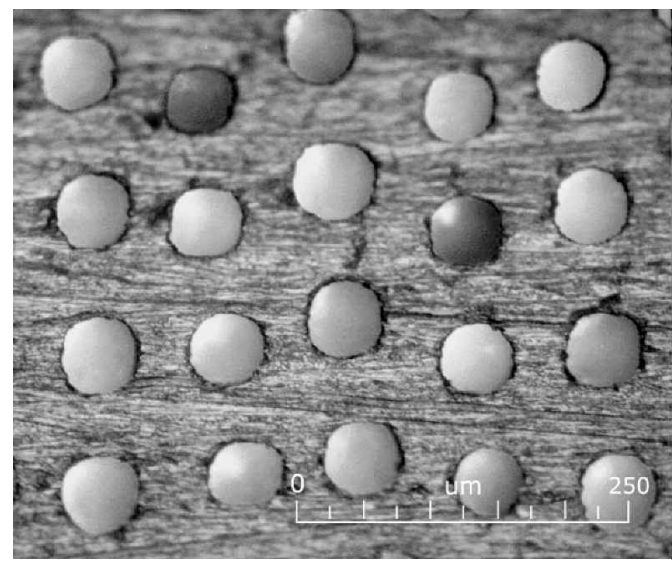

b)

Fig. 2 Magnified image of a) weld joining the porous and solid sheets and b) stainless-steel perforated sheet. 


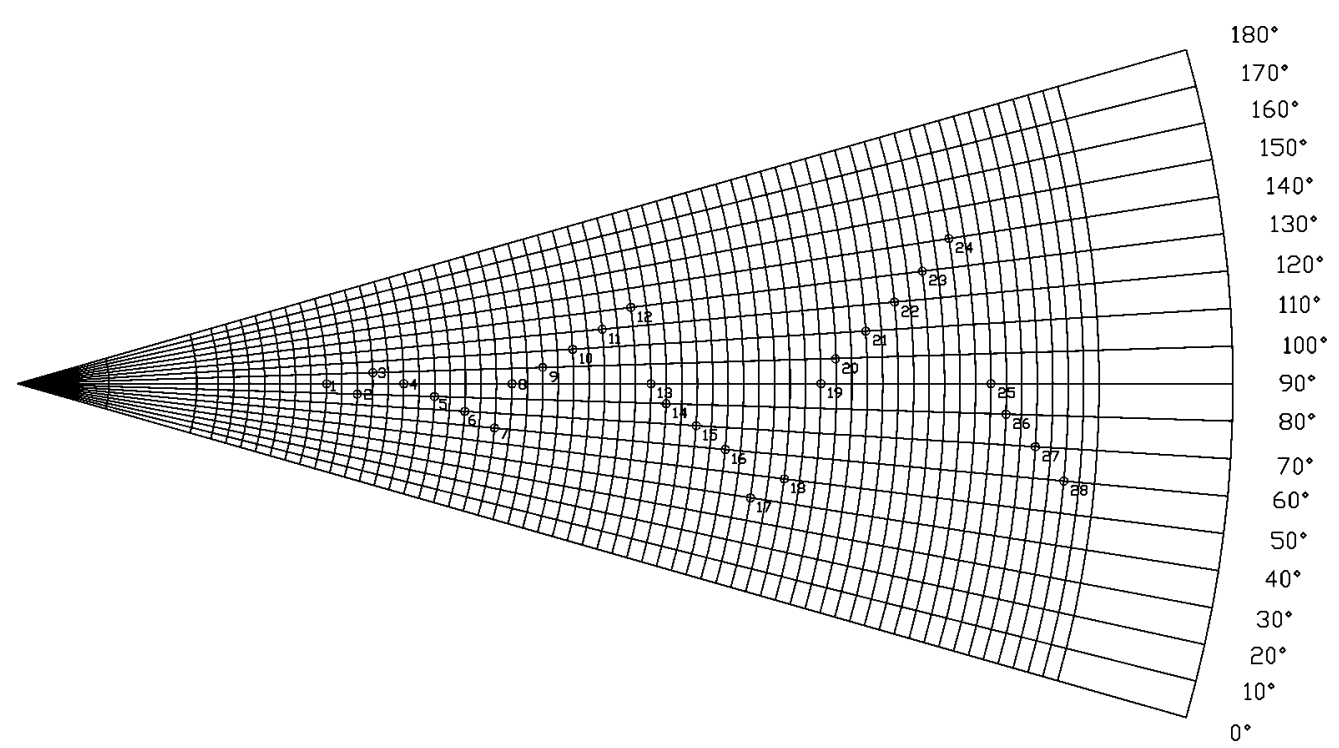

Fig. 3 Schematic diagram showing the locations of the thermocouples on a developed view of half the cone.

placed along the 40-deg ray. This is deemed more than adequate because shock tunnel experiments by $\mathrm{Me}^{13}$ in Mach 5 hypervelocity flow over a flat plate indicated that the spreading half-angle of turbulent bursts is approximately $3-4 \mathrm{deg}$.

The small (0.8-mm-diam) and fast-response (1- $\mu \mathrm{s})$ thermocouples were manufactured in-house based on a modified design originally developed by Sanderson ${ }^{14}$ and whose performance was tested in detail by Davis. ${ }^{15}$ During the shot, the thermocouple signal was amplified by a factor of 500 and then sampled at $200 \mathrm{kHz}$. The sampled voltage levels were converted to temperature using correlations for Type E thermocouples. The heat flux for each thermocouple was subsequently computed using a spectral deconvolution technique ${ }^{14-16}$ based on the heat equation for one-dimensional unsteady heat transfer in a semi-infinite solid.

\section{Results}

A total of 29 shots were carried out in nitrogen, and 19 shots were performed in carbon dioxide. The nitrogen shots were performed with reservoir pressures ranging from 11 to $50 \mathrm{MPa}$ and specific reservoir enthalpies ranging from 3.0 to $13 \mathrm{MJ} / \mathrm{kg}$. Above $13 \mathrm{MJ} / \mathrm{kg}$, the Reynolds numbers achieved in T5 were too low to observe transition on the cone. The carbon dioxide shots were performed with reservoir pressures ranging from 9.0 to $40 \mathrm{MPa}$ with specific reservoir enthalpies ranging from 1.3 to $9.0 \mathrm{MJ} / \mathrm{kg}$. The complete set of data and results is available. ${ }^{16}$

\section{Transition Reynolds Number}

The transition Reynolds number was determined by examining the heat transfer traces measured at each thermocouple station. For each shot, a heat flux level for each thermocouple was obtained by averaging over a short time period after the nozzle starting process but before the onset of driver gas contamination and always within the constant reservoir pressure window.

The heat flux levels were used to produce nondimensionalplots of Stanton number vs Reynolds number (evaluated at edge conditions) such as in Fig. 4. The Stanton number was computed as

$$
S t=\frac{\dot{q}(x)}{\rho_{e} U_{e} h_{\mathrm{aw}}}
$$

where $\dot{q}$ is the heat transfer rate, $\rho_{e}$ is the edge density, $U_{e}$ is the edge velocity, and $h_{\text {aw }}$ is the enthalpy assuming an adiabatic wall. The Reynolds number was computed as

$$
\operatorname{Re}_{x}=\rho_{e} U_{e} x / \mu_{e}
$$

where $\rho_{e}$ and $U_{e}$ are as before, $x$ is the distance along the surface of the cone, and $\mu_{e}$ is the viscosity evaluated at the edge conditions. The viscosity was calculated using a simple code based on a viscosity model for reacting gases developed by Blottner et al. ${ }^{17}$ to determine the viscosity of each species in the gas mixture at the edge temperature. Coefficients for the model for the different gases were obtained from Olynick et al. ${ }^{18}$ When the computed gas composition at the edge condition was used, the code then used Wilke's ${ }^{19}$ semi-empirical mixing rule to calculate the overall viscosity of the gas mixture.

In Figs. 4a-4f, each point represents a heat flux value (or Stanton number $S t$ ) for the thermocouple at that particular location (or Reynolds number $R e_{x}$ ). The dark black data points correspond to the data collected in the current series of experiments, whereas the gray data points correspond to previous results. The state of the boundary layer was determined by comparing the experimental results with theoretical models assuming frozen chemistry. The solid line that essentially runs through the data points represents the theoretical value for a frozen, noncatalytic surface laminar boundary layer. The dashed-dotted and dotted lines represent the expected values for turbulent boundary layer as computed using semi-empirical models developed by Van Driest and White/Christoph, respectively. These models are described in detail by White, ${ }^{20}$ Adam, ${ }^{12}$ and Rasheed. ${ }^{16}$ Although error bars are not shown in Figs. $4 \mathrm{a}-4 \mathrm{f}$ for clarity, the uncertainty in the Stanton number was computed based on the uncertainty in the dimensional heat flux (from \pm 13 to $\pm 18 \%$ ), the stagnation enthalpy $( \pm 8 \%)$, the edge velocity $( \pm 4 \%)$, and the edge density $( \pm 8 \%)$. The details of the estimation of these uncertainties are presented by Rasheed. ${ }^{16}$ The final uncertainty in the Stanton number for each thermocouple ranged from about \pm 18 to $\pm 22 \%$.

The transition location was determined by first fitting a line through the data points in the laminar region (the dashed-triple dotted line near the solid line) while enforcing the Reynolds number $R e^{-0.5}$ law expected for a zero pressure gradient laminar boundarylayer. Another line was then fitted through the data points in the transition region (the other dashed-triple dotted line). The intersection of these two lines was determined to be the transition Reynolds number, that is, the onset of transition. Note that, although including fewer or more data points in the curve fit for the transition region changes the slope of the dashed-triple dotted line, it does not significantly change the intersection with the laminar line. The uncertainty in the transition Reynolds number was computed by first determining upper and lower uncertainty bounds for the laminar experimental data fit from the linear regression assuming the 95\% confidence level using the small sample T distribution. Although not shown in Figs. 4a-4f for clarity, these upper and lower uncertaintybounds would be represented as parallel lines above and below the laminar experimental fit. Similarly, another pair of lines was drawn above and below the transitional experimental fit. The intersections of these two sets of upper and lower bounds formed an error rhombus around the transition location. The minimum and 

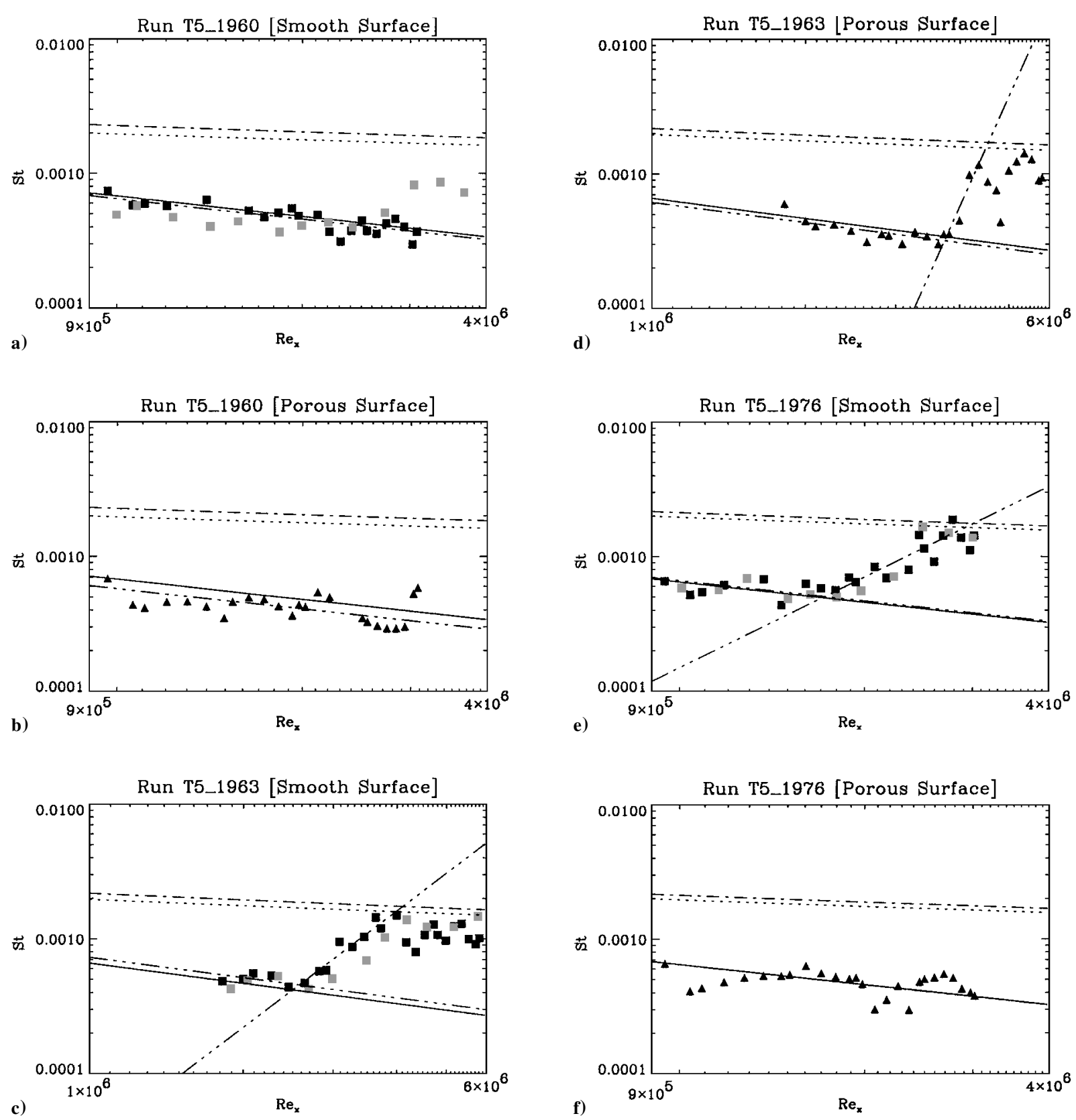

Fig. 4 Stanton number $S t$ vs Reynolds number $R e_{x}$.

maximum vertices (in terms of absissca coordinates) of the error rhombus were used as the upper and lower uncertainty bounds, and the average percent error of these two values was used as the estimate for the uncertainty in the transition location. This uncertainty varied from shot to shot, but it ranged from \pm 3 to $\pm 15 \%$, depending on the slope of the transitional fit. When the additional uncertainty due to density $( \pm 8 \%)$, velocity $( \pm 4 \%)$ and viscosity $( \pm 5 \%)$ were factored in, the overall uncertainty in transition Reynolds number ranged from \pm 11 to $\pm 19 \%$, with a median of $\pm 13 \%$.

\section{Case 1: Both Sides Laminar}

Figures $4 \mathrm{a}$ and $4 \mathrm{~b}$ show plots of Stanton number $S t$ vs Reynolds number $R e_{x}$ obtained from shot 1960, a high-enthalpy shot ( $p_{0}=$ $51.5 \mathrm{MPa}, h_{0}=12.8 \mathrm{MJ} / \mathrm{kg}$ ) in nitrogen. Figures $4 \mathrm{a}$ and $4 \mathrm{~b}$ show that the boundary layer was laminar over the entire length of the cone for both the smooth and porous surfaces. Furthermore, the results obtained on the smooth surface are shown to be in excellent agreement with previous results obtained by Germain and Hornung ${ }^{1}$ for essentially the same run condition. Note that the Ref. 1 data indicate that the boundarylayer is just beginning to transition toward the back of the model, whereas this effect is not evidentin the present experiment. This is attributed to the slightly higher unit Reynolds number obtained in Germain and Hornung's experiments.

\section{Case 2: Both Sides Transitional}

Figures $4 \mathrm{c}$ and $4 \mathrm{~d}$ show plots of Stanton number $S t$ vs Reynolds number $R e_{x}$ for shot 1963 , which was a midenthalpy shot $\left(p_{0}=\right.$ $45.6 \mathrm{MPa}, h_{0}=7.2 \mathrm{MJ} / \mathrm{kg}$ ) in nitrogen. Figures $4 \mathrm{c}$ and $4 \mathrm{~d}$ show typical transition behavior with the data following the theoretical laminar curve at low Reynolds number and moving to the expected turbulent curve farther downstream. Figures $4 \mathrm{c}$ and $4 \mathrm{~d}$ show that the boundary layer on the smooth surface transitions well upstream as compared to the porous surface and appears to validate the prediction by Fedorov et al. ${ }^{10}$ Once again, the Germain and Hornung ${ }^{1}$ data for a similar run condition is shown for comparison with excellent agreement as to the transition location.

\section{Case 3: Porous Side Laminar, Smooth Side Transitional}

Figures $4 \mathrm{e}$ and $4 \mathrm{f}$ show plots of Stanton number $S t$ vs Reynolds number $R e_{x}$ obtained from shot 1976, a low-enthalpy shot $\left(p_{0}=\right.$ 14.5 MPa, $h_{0}=5.0 \mathrm{MJ} / \mathrm{kg}$ ) in nitrogen. In this particular case, the smooth surface boundary layer transitions roughly at the halfway 
point on the cone whereas the porous sheet boundarylayer is laminar all of the way to the end of the cone. This shot clearly demonstrates the dramatic effect of the porous surface in delaying boundary-layer transition. Once again, notice the good agreement with the previous experiment performed by Germain and Hornung. ${ }^{1}$

\section{Summary Data}

The summary data are presented in the form of plots of Reynolds number $R e_{\mathrm{tr}}^{*}$ vs stagnation enthalpy $h_{0}$ (Figs. 5 and 6). The data are presented in this manner because both Germain and Hornung ${ }^{1}$ and Adam and Hornung ${ }^{2}$ found a correlation of Reynolds number $R e_{\mathrm{tr}}^{*}$ with stagnation enthalpy $h_{0}$, where the transition Reynolds number (evaluated at reference conditions) is calculated as

$$
R e_{\mathrm{tr}}^{*}=\rho^{*} U_{e} x_{\mathrm{tr}} / \mu^{*}
$$

where $\rho^{*}$ is the density evaluated at the reference condition, $U_{e}$ is the edge velocity, $x_{\text {tr }}$ is the transition location (as defined earlier) measured along the surface of the cone, and $\mu^{*}$ is the viscosity evaluated at the reference condition. In addition, there was reason to believe that the effectiveness of the porous surface would depend on $h_{0}$ because calculations by Johnson et al. ${ }^{7}$ in air indicated that the most unstable mode frequencies varied with $h_{0}$.

\section{Nitrogen Shots}

Figure 5 gives a summary of the data for the nitrogen shots. The first observation is that the present experimental results (dark data points) agree fairly well with the previous results obtained by Germain and Hornung ${ }^{1}$ (gray data points). The diamonds represent the $R e_{\text {tr }}^{*}$ on the porous surface, whereas the squares represent the $R e_{\text {tr }}^{*}$ on the smooth surface for the same shot. The second observation is that, in all cases, the porous surface delayed transition by a significant amount. The open diamonds with an upward-facing arrow $\uparrow$ indicate that the porous surface boundary layer was laminar to the very end of the cone. The value plotted assumes that transition occurred at the last thermocouple, that is, unit Reynolds number

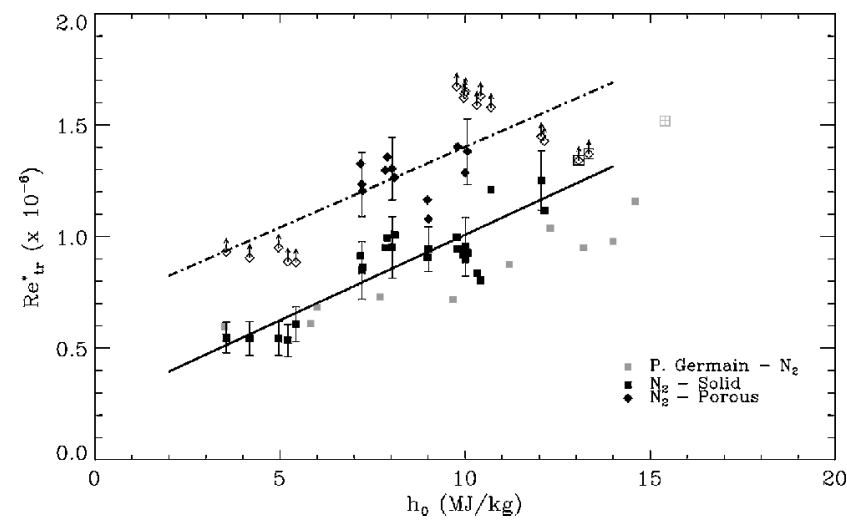

Fig. 5 Reynolds number $R e_{\mathrm{tr}}^{*}$ vs $h_{0}$ summary of nitrogen data.

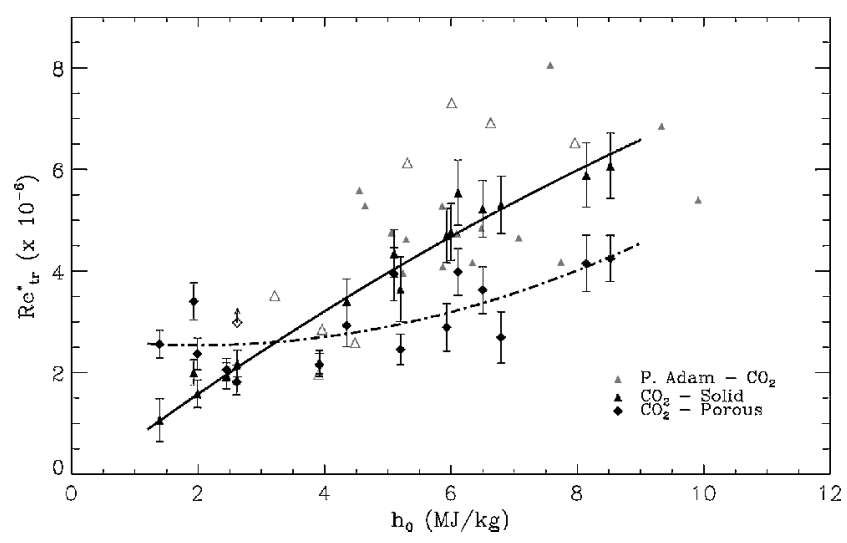

Fig. 6 Reynolds number $R e_{\mathrm{tr}}^{*}$ vs $h_{0}$ summary of the carbon dioxide data. multiplied by the last thermocouple location. This is not a valid data point but rather a manner in which to show that the boundary layer was entirely laminar. The same discussion applies for the open square symbols with an $\uparrow$ for the smooth surface side. The two data points at $13 \mathrm{MJ} / \mathrm{kg}$ are actually open diamonds superimposed on open squares, with an $\uparrow$, indicating that both the smooth and porous surface boundary layers were entirely laminar. The lines are linear curve fits to help guide the eye (dashed-dotted for porous and solid for smooth). Note that the porous sheet appears to be less effective at midenthalpies as compared to low and high enthalpies.

\section{Carbon Dioxide Shots}

An analysis similar to that performed for the nitrogen shots was also performed for each carbon dioxide shot. The resulting summary plot of Reynolds number $R e_{\mathrm{tr}}^{*} \mathrm{vs} h_{0}$ is shown in Fig. 6. Once again there is fairly good agreement between the present smooth surface results (dark data points) and those obtained in previous experiments by Adam and Hornung ${ }^{2}$ (gray data points), although there is much more scatter. Figure 6 shows that the porous sheet (diamonds) was, in fact, detrimental at higher enthalpies, but was effective at lower enthalpies with a crossover point at roughly $3.0 \mathrm{MJ} / \mathrm{kg}$. The diamonds represent the $R e_{\mathrm{tr}}^{*}$ on the porous surface, whereas the triangles represent Reynolds number $R e_{\mathrm{tr}}^{*}$ on the smooth surface for the same shot. The lines are second-order curve fits to help guide the eye (dash-dotted for porous and solid for smooth).

\section{Resonantly Enhanced Shadowgraph}

Further evidence of the effectiveness of the porous sheet is seen in Fig. 7, which is a resonantly enhanced shadowgraph showing the boundary-layertransitioning on the smooth surface (top) while remaining perfectly laminar on the porous surface (bottom). Flow is left to right and the schematic at the top right indicates the window position relative to the model. The rectangular boxes in the main image indicate the location of the magnified images whose left and right edges are 495 and $615 \mathrm{~mm}$ from the cone tip, respectively (as measured along the surface of the cone). The white line on the magnified image of the smooth surface was digitally added to indicate the approximate surface of the model. This shadowgraph corresponds to shot $2008\left(P_{0}=48.2 \mathrm{MPa}, h_{0}=9.8 \mathrm{MJ} / \mathrm{kg}\right)$ and was obtained by seeding the flow with sodium and tuning the frequency of the dye laser light source to one of the sodium D lines. The transition location on the smooth surface identified by the analysis of the heat transfer data is approximately at the left edge of the magnified image of the smooth surface. The magnified images were also used to measure the boundary-layer thickness, and this value was found to be approximately $1 \mathrm{~mm}$, which is in agreement with Adam's computations. ${ }^{12}$

\section{Discussion}

As already indicated, to validate the effectiveness of the porous surface, it was necessary to ensure that angle-of-attack or nonaxisymmetry issues were not affecting the results and that the results were repeatable. Angle of attack was eliminated as a cause for the delayed transition by carefully aligning the model to within $\pm 0.05 \mathrm{deg}$ of the tunnel axis. Furthermore, it was noted that the observed effect was to delay transition as much as $100 \%$ (or more because the cone was not long enough). This is significantly larger than the $5 \%$ variation in Reynolds number $R e_{\text {tr }}$ that could be attributed to an angle of attack of 0.1 deg as observed by Krogmann (and reported by Stetson ${ }^{21}$ ) in his study of the effects of angle of attack on Reynolds number $R e_{\text {tr }}$ in hypersonic flow $\left(M_{\infty} \simeq 5\right)$ over a 5-deg half-angle cone.

Flow asymmetry issues were addressed by rotating the model to the $0-, 90-$, and 180-deg orientations and repeating the experiments. In particular, two run conditions were repeated and can be seen in Fig. 5 as the data points clustered at 5 and $8 \mathrm{MJ} / \mathrm{kg}$, respectively. Clearly, there is excellent agreement, and there appears to be no effects from rotating the model. The model asymmetries due to imperfections were discussed earlier and were determined to be irrelevant in the context of the present results.

Repeatability was tested by repeating selected experimental run conditions at various stages in the test program and noting that there 


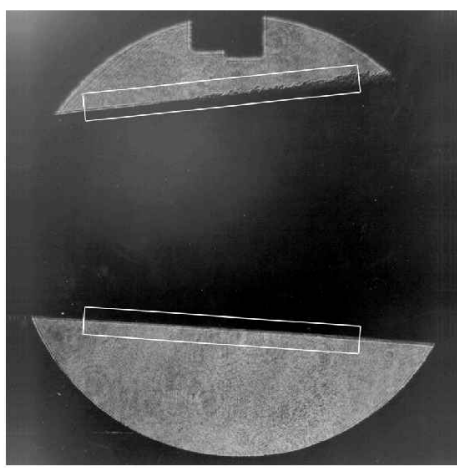

Smooth Surface

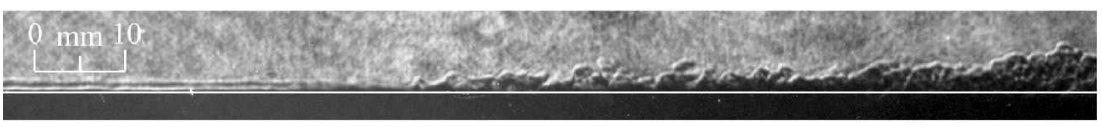

Porous Surface

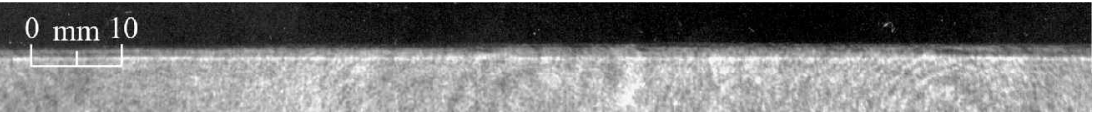

Fig. 7 Resonantly enhanced shadowgraph.

was no observable effect on transition location. This was of particular importance because it confirmed that the slight accumulation of soot on the surface of the model over the course of the experiments had no effect on the results. The soot was generated by the pyrolization of the polyurethanebuffers used to stop the piston in this facility and was carried by the driver gas at the end of each shot, that is, the soot arrived at the model after the useful test time. Extensive testing performed on two sample test pieces of the porous surface mounted on Plexiglas ${ }^{\circledR}$ backings (flat plate normal to the flow and cylinder inclined at $5 \mathrm{deg}$ to the flow) before testing the actual cone model had previously suggested that the soot would not be a problem. The Plexiglas mountings allowed the samples to be backlit so that it was possible to determine whether the holes were clogged (no light passing through). Based on these tests it was decided to allow the soot to accumulate over the course of the series of experiments. The repeatability tests confirmed that the soot had no effect on the results and also provided an indication of the robustness of this boundary-layercontrol scheme to small amounts of contamination.

As already mentioned, for the nitrogen experiments, the porous surface was effective over the whole enthalpy range tested, although it was more effective at low- and high-enthalpy conditions than at midenthalpy conditions. For the carbon dioxide shots, however, the porous surface was only effective at very low-enthalpy conditions and was counterproductive at mid- to high enthalpies. This behavior suggested that another parameter was important in the carbon dioxide flows.

A possible explanation for the observed behavior lies in the expectation that the porous surface must be hydraulically smooth, that is, pore size must be sufficiently small in relation to the viscous length scale, for the proposed mechanism to effectively delay transition. If this were not the case, then the holes would act as distributed surface roughness and prematurely trip the boundary layer. A plot of $R e_{\mathrm{tr} \text {,porous }}^{*}-R e_{\mathrm{tr}, \text { smooth }}^{*} \mathrm{vs}$ Reynolds number based on pore diameter $R e_{D}^{*}$ clearly shows that the delay in transition becomes much smaller as Reynolds number $R e_{D}^{*}$ increases (Fig. 8). Note that no such trend was observed when the results were plotted using the Reynolds number evaluated at the edge conditions $\left(R e_{D}\right)$, and it is, therefore, concluded that the reference Reynolds number is the proper Reynolds number to use in this analysis for these flows. The grayscale indicates the qualitative effectiveness of the porous surface. Black indicates laminar over the entire length of the cone on the porous side, medium gray indicates delayed transition was observed on the cone, and light gray indicates premature transition. Squares and triangles correspond to $\mathrm{N}_{2}$ and $\mathrm{CO}_{2}$, respectively. Figure 8 shows that the mechanism becomes qualitatively less effective at an Reynolds number $R e_{D}^{*}$ of about 130 and actually prematurely trips the boundary layer at an Reynolds number

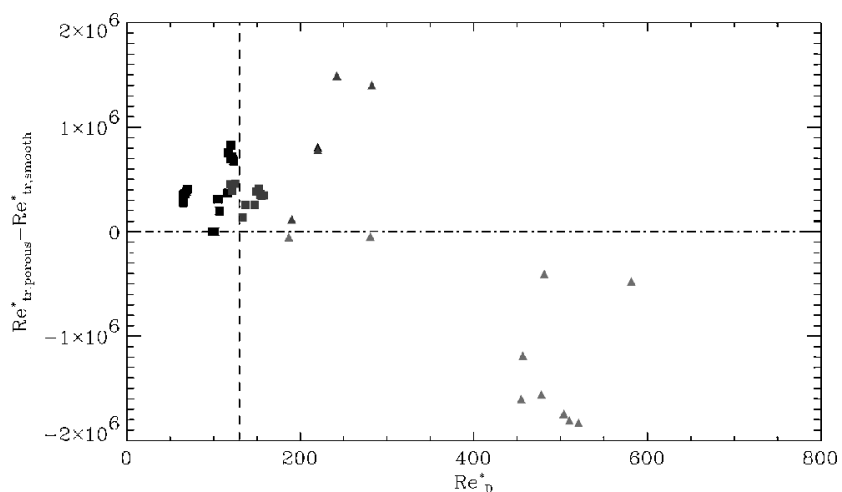

Fig. 8 Decreased effectiveness as Reynolds number $R e_{D}^{*}$ increases beyond 130 (vertical line).

$R e_{D}^{*}$ greater than 300 . The most relevant experiment (to the present case) regarding distributed surface roughness effects was performed by Germain and Hornung, ${ }^{1}$ who found that $0.1-\mathrm{mm}$ salt crystals $\left(R e_{k}^{*}=220\right.$, based on salt crystal height) randomly distributed over the first $206 \mathrm{~mm}$ of the model tripped the boundary layer. No attempt was made to identify the critical roughness Reynolds number below which the surface roughness had no effect on transition. For such a comparison, it is useful to recognize that the present results correspond reasonably well with experiments in incompressible flow by Feindt (as reported by Schlichting ${ }^{22}$ ), who examined the effect of distributed surface roughness (in the form of sand grains) on transition Reynolds number. Although the type of roughness differed from the present experiments (sand grains vs porous surface), Feindt also found that the surface roughness became important when Reynolds number $\operatorname{Re}_{k}$ (based on the sand grain height) was greater than 120. Similarly, Pfenninger ${ }^{23}$ reported that surface roughness issues were important in laminar flow control experiments (suction through slots) when Reynolds number $R_{s}$ (based on slot width) was approximately greater than 100 .

More recently, $\operatorname{Reda}^{24}$ reviewed the effects of distributed surface roughness in hypervelocity flows on nose tips, attachment lines, and lifting entry vehicles. Reda concluded that there exists no universal value for critical Reynolds number $R e_{k}$ (based on roughness height) for transition to turbulence and that this critical roughness Reynolds number was highly dependent on the particular flowfield and roughness characteristics. Despite this observation, Reda indicated that a number of different experiments suggested that the critical roughness Reynolds number ranges between 100 and 200. Reda's earlier experiments on nose tip transition in a ballistic range yielded values 


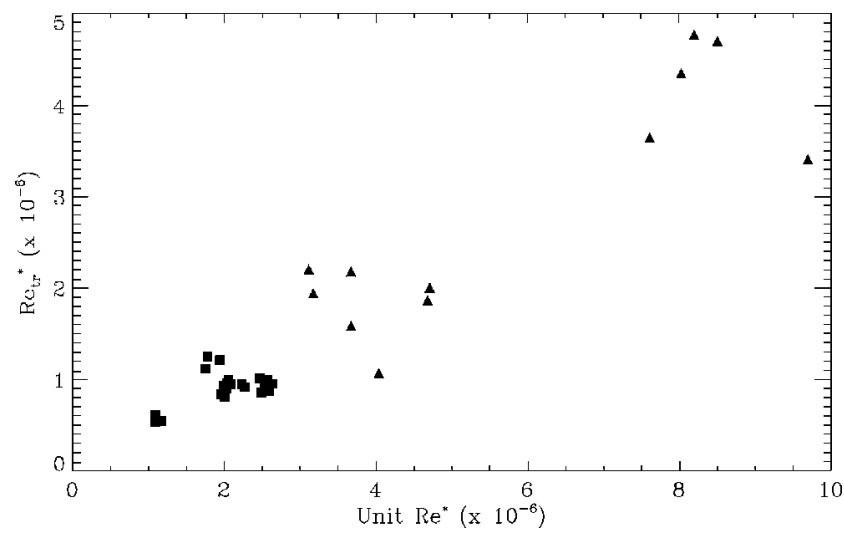

Fig. 9 Reynolds number $R e_{\mathrm{tr}}^{*}$ vs Unit $R e^{*}$ showing unit Reynolds number effect observed in the transition data on the smooth surface.

for the critical Reynolds number $R e_{k}$ of 192 . Bertin et al. (as reported by $\operatorname{Reda}^{24}$ ) found $R e_{k}=110$ to be the critical value at which roughness effects began to dominate in wind-tunnel tests of a 0.0175 scale model of the Space Shuttle Orbiter at Mach 8-12. Finally, Goodrich et al. (as reported by $\operatorname{Reda}^{24}$ ) found that the transition data on the windward centerline of the Space Shuttle Orbiter during reentry for missions STS- 1 to STS-5 suggested a critical Reynolds number $R e_{k}$ of 120 . Note that in these cases, the Reynolds number $R e_{k}$ value was for surface bumps (as opposed to holes in the present work) and were calculated using the conditions in the boundary layer at the roughness height. This may or may not allow direct comparison with the Reynolds number $R e_{D}^{*}$ used to analyze the present results, but it is clear that the critical Reynolds number $R e_{D}^{*}$ is in the same range as previous experiments.

Note, however, that closer examination of Fig. 8 also indicates that some parameter is still not fully accountedfor because the effectiveness of the porous sheet does not decrease monotonically with increasing Reynolds number $R e_{D}^{*}$. Specifically, the porous sheet appeared to be more effective in the carbon dioxide shots at $R e_{D}^{*} \simeq 200$ than the nitrogen shots at $R e_{D}^{*} \simeq 130$. Although unexpected, it is not entirely surprising that nitrogen and carbon dioxide behave slightly differently because the different chemistry involved could affect the second mode amplification.

A final interesting observation is noted if one examines the socalled unit Reynolds number effect. Figure 9 is a plot of Reynolds number $R e_{\mathrm{tr}}^{*}$ vs Unit $R e^{*}$ for the smooth surface (squares and triangles correspond to $\mathrm{N}_{2}$ and $\mathrm{CO}_{2}$, respectively). As expected, it shows the typically strong dependence of transition Reynolds number on unit Reynolds number. Of course, the dependence of a nondimensional quantity on a dimensional quantity simply means that there is another factor that has not been taken into account. In the case of transition data, this other factor is typically associated with the acoustic noise generated by the nozzle wall boundary layer, as was shown by Pate and Schueler. ${ }^{25}$ Note that, as before, this trend is not observed in the present data if one plots the Reynolds number based on edge conditions (as opposed to the reference conditions). This seems to further support the conclusion by Germain and Hornung ${ }^{1}$ and Adam and Hornung ${ }^{2}$ that the reference conditions are the appropriate conditions to use when evaluating these flows. An even more interesting observation is noted if one examines a similar plot for the porous surface as shown in Fig. 10, where squares and triangles correspond to $\mathrm{N}_{2}$ and $\mathrm{CO}_{2}$, respectively. In this case, there is no observed unit Reynolds number effect (when plotted using either edge conditions or reference conditions). At this time, it is unclear why this would be the case. A very reasonable explanation, however, is that the effect of the porous surface is to damp out the second mode acoustic perturbations. As such, it definitely also attenuates the acoustic noise from the nozzle wall boundary layer, and it is entirely possible that the porous surface is so effective that it completely removes the unit Reynolds number effect. Such a conclusion would have very far-reaching implications and would need to be considered very carefully in much more detail in the future.

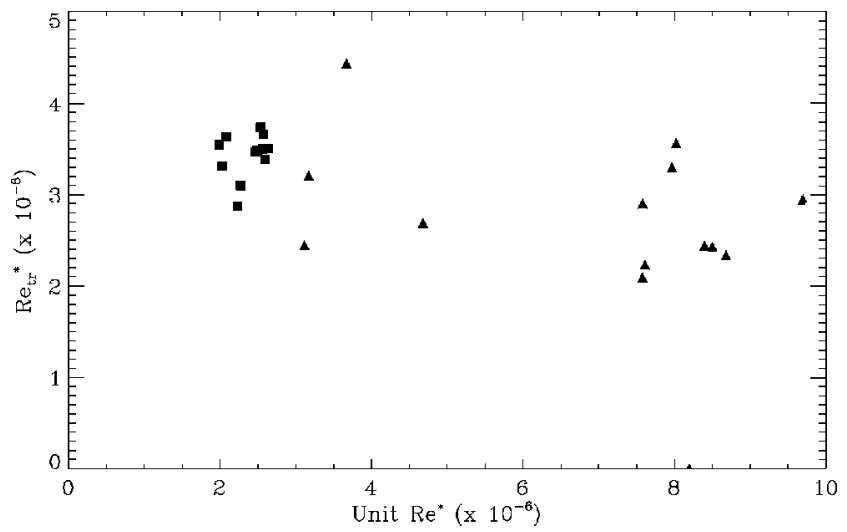

Fig. 10 Reynolds number $R e_{\mathrm{tr}}^{*}$ vs Unit $R e^{*}$ showing that unit Reynolds number effect was not observed in the transition data on the porous surface.

\section{Conclusions}

Extensive experiments on hypervelocity boundary-layer transition control have been carried out in the T5 on a 5.06-deg half-angle sharp cone with a smooth surface over half the cone and an ultrasonically absorptive porous surface over the other half. These experiments, performed in nitrogen and carbon dioxide, used heat transfer measurements to determine simultaneously the transition location on the smooth and porous surfaces for each shot. These new measurements for the smooth surface transition location compared very well with experimentalresults obtained by previousresearchers in the same facility. The theoretical result that transition may be delayed by suitable wall porosity has been confirmed convincingly in nitrogen flows, and the reversal of the phenomenon in carbon dioxide flows appears to be due to the chosen wall porosity scale being too coarse in carbon dioxide. These results were tested for repeatability and were checked to ensure that they were not induced by angle-of-attack or other effects. The effectiveness of the porous sheet was further evidenced by a resonantly enhanced shadowgraph that clearly showed transition occurring over the smooth surface but not the porous surface.

\section{Acknowledgment}

This work was sponsored by the Air Force Office of Scientific Research (AFOSR), U.S. Air Force, under AFOSR Grant F4962098-1-0353 (Steven Walker).

\section{References}

${ }^{1}$ Germain, P., and Hornung, H. G., "Transition on a Slender Cone in Hypervelocity Flow," Experiments in Fluids, Vol. 22, No. 3, 1997, pp. 183190.

${ }^{2}$ Adam, P., and Hornung, H. G., "Enthalpy Effects on Hypervelocity Boundary Layer Transition: Ground Test and Flight Data,' Journal of Spacecraft and Rockets, Vol. 34, No. 5, 1997, pp. 614-619.

${ }^{3}$ Eckert, E. R. G., "Engineering Relations for Friction and Heat Transfer to Surfaces in High Velocity Flow," Journal of the Aeronautical Sciences, Vol. 22, Aug. 1955, pp. 585-587.

${ }^{4}$ Stetson, K. F., "Hypersonic Boundary-Layer Transition," Advances in Hypersonics: Volume I, Defining the Environment, edited by J. J. Bertin, J. Periaux, and J. Ballmann, Birkhäuser, Boston, 1992, pp. 324-417.

${ }^{5}$ Mack, L. M., "Boundary-Layer Stability Theory," Special Course on Stability and Transition of Laminar Flow, Rept. 709, AGARD, 1984.

${ }^{6}$ Stetson, K. F., Thompson, E. R., Donaldson, J. C., and Siler, L. G., "Laminar Boundary Layer Stability Experiments on a Cone at Mach 8, Part 1: Sharp Cone," AIAA Paper 83-1761, July 1983.

${ }^{7}$ Johnson, H. B., Seipp, T., and Candler, G. V., "Numerical Study of Hypersonic Reacting Boundary Layer Transition on Cones," Physics of Fluids, Vol. 10, No. 10, 1998, pp. 2676-2685.

${ }^{8}$ Fedorov, A. V., and Malmuth, N. D., "Hypersonic Flow Stabilization by Ultrasonically Transparent Wall," Rockwell Science Center, TR SCNM96-1, Thousand Oaks, CA, 1996.

${ }^{9}$ Fedorov, A. V., Malmuth, N. D., Shalaev, V., Cole, J., and Khokhlov, A., "Problems in High Speed Flow Prediction Relevant to Control," AIAA Paper 98-2995, June 1998.

${ }^{10}$ Fedorov, A. V., Malmuth, N. D., Rasheed, A., and Hornung, H. G., "Stabilization of Hypersonic Boundary Layers by Porous Coatings," AIAA Journal, Vol. 39, No. 4, 2001, pp. 605-610. 
${ }^{11}$ Hornung, H. G., "Performance Data of the New Free-Piston Shock Tunnel T5 at GALCIT," AIAA Paper 92-3943, July 1992.

${ }^{12}$ Adam, P., "Enthalpy Effects on Hypervelocity Boundary Layers," Ph.D. Dissertation, California Inst. of Technology, Pasadena, CA, 1997.

${ }^{13}$ Mee, D. J., "Boundary Layer Transition Measurements in Hypervelocity Flows in a Shock Tunnel," AIAA Paper 2001-0208, Jan. 2001.

${ }^{14}$ Sanderson, S. R., "Shock Wave Interaction in Hypervelocity Flow," Ph.D. Dissertation, California Inst. of Technology, Pasadena, CA, 1995.

${ }^{15}$ Davis, J. P., "High-Enthalpy Shock/Boundary-Layer Interaction on a Double Wedge," Ph.D. Dissertation, California Inst. of Technology, Pasadena, CA, 1999.

${ }^{16}$ Rasheed, A., "Passive Hypervelocity Boundary Layer Control Using an Ultrasonically Absorptive Surface," Ph.D. Dissertation, California Inst. of Technology, Pasadena, CA, 2001.

${ }^{17}$ Blottner, F. G., Johnson, M., and Ellis, M. G., "Chemically Reacting Viscous Flow Program for Multi-Component Gas Mixtures," Sandia Lab., TR SC-RR-70-754, Albuquerque, NM, Dec. 1971.

${ }^{18}$ Olynick, D., Chen, Y.-K., and Tauber, M., "Aerothermodynamics of the Stardust Sample Return Capsule," Journal of Spacecraft and Rockets, Vol. 36, No. 3, 1999, pp. 442-462.
${ }^{19}$ Wilke, C. R., "A Viscosity Equation for Gas Mixtures," Journal of Chemical Physics, Vol. 18, No. 4, 1950, pp. 517-519.

${ }^{20}$ White, F. M., Viscous Fluid Flow, 2nd ed., McGraw-Hill, New York, 1991, pp. 549-554.

${ }^{21}$ Stetson, K. F., "Mach 6 Experiments of Transition on a Cone at Angle of Attack," Journal of Spacecraft and Rockets, Vol. 19, No. 5, 1982, pp. 397403.

${ }^{22}$ Schlichting, H., Boundary Layer Theory, McGraw-Hill, New York, 1987, Chap. 17, Sec. g, p. 542.

${ }^{23}$ Pfenninger, W., "Laminar Flow Control," Special Course on Concepts for Drag Reduction, Rept. 654, AGARD, 1977.

${ }^{24}$ Reda, D. C., "Roughness-Dominated Transition on Nosetips, Attachment Lines and Lifting-Entry Vehicles," AIAA Paper 2001-0205,Jan. 2001.

${ }^{25}$ Pate, S. R., and Schueler, C. J., "Radiated Aerodynamic Noise Effects on Boundary-Layer Transition in Supersonic and Hypersonic Wind Tunnels," AIAA Journal, Vol. 7, No. 2, 1969, pp. 450-457.

W. J. Devenport Associate Editor 